\title{
Hercules X-1: variations of the cyclotron line energy with flux, with phase and with time
}

\author{
Rüdiger Staubert ${ }^{* \dagger}$ \\ Institut für Astronomie und Astrophysik, Universität Tübingen, Sand 1, D-72076 Tübingen, \\ Germany \\ E-mail: stauberteastro.uni-tuebingen.de
}

Her X-1 is one of the the most intensively studied Accreting X-ray Binary Pulsars (AXBPs). This is largely because it is a bright and persistent X-ray pulsar, showing the largest variety of observable phenomena, partly due to the favorable geometry in observing the binary nearly edgeon. Her X-1 is the binary X-ray pulsar in which the first cyclotron line (or Cyclotron Resonant Scattering Feature - CRSF) was detected in 1976. Since then, we have made an effort to observe the source and the CRSF around $40 \mathrm{keV}$ as often as possible, using those X-ray satellites which cover the high energy X-ray range. Extended contributions are from the Rossi $X$-ray Timing Explorer (RXTE), INTEGRAL, Suzaku and Swift. The most recent contribution with a new degree of quality is from the high energy imaging telescope on NuStar. We have found that the CRSF is variable with flux, with phase (both pulsational and precessional phase) and with time. The historical evolution of the pulse phase averaged CRSF centroid energy $\mathrm{E}_{\text {cyc }}$ since its discovery is characterized by an initial value around $35 \mathrm{keV}$, an abrupt jump upwards to beyond $\sim 40 \mathrm{keV}$ between 1990 and 1994 and an apparent secular decay thereafter. Much of this decay, however, was found to be due to an artifact, namely a correlation between $\mathrm{E}_{\mathrm{cyc}}$ and the X-ray luminosity $L_{x}$ discovered in 2007, amounting to a change of $\sim 7 \%$ in energy for a factor of two in luminosity. In observations since 2007 , however, we now find a statistically significant trend of a true secular decrease of the cyclotron line energy. In addition, we discuss recent results of pulse phase spectroscopy and evidence for the suspected variation in $E_{\text {cyc }}$ with phase of the $35 \mathrm{~d}$ precessional period (which is observed both in the modulation of the X-ray flux and in the systematic variation in shape of the $1.24 \mathrm{~s}$ pulse profile).

"An INTEGRAL view of the high-energy sky (the first 10 years)" 9th INTEGRAL Workshop and celebration of the 10th anniversary of the launch,

October 15-19, 2012

Bibliotheque Nationale de France, Paris, France

\footnotetext{
*Speaker.

${ }^{\dagger}$ with D. Klochkov, D. Vasco, K. Postnov, N. Shakura, J. Wilms, R. Rothschild, A. Santangelo, B. Grefenstette, F. Fürst, F. Harrison and others
} 
Table 1: Details of recent observations of Her X-1 by INTEGRAL, RXTE Suzaku and NuStar.

\begin{tabular}{llllc}
\hline Observatory & $\begin{array}{l}\text { Date of } \\
\text { observation }\end{array}$ & $\begin{array}{l}\text { Center } \\
\text { MJD }\end{array}$ & Obs ID & $\begin{array}{l}\text { Exposure } \\
{[\mathrm{ksec}]}\end{array}$ \\
\hline INTEGRAL & 2007 Sep 03-08 & 54348.86 & Rev. 597/598 & 414.72 \\
RXTE & 2009 Feb 04/05 & 54866.95 & P 80015 & 22.19 \\
Suzaku & 2010 Sep 22 & 55461.63 & $405058010 / 20$ & 41.66 \\
Suzaku & 2010 Sep 29 & 55468.51 & $405058030 / 40$ & 45.66 \\
INTEGRAL & 2010 July 10-18 & 55391.43 & Rev. 945-947 & 621.03 \\
Suzaku & 2012 Sep 19-25 & 56192.23 & $4070510-10,20,30$ & $\sim 70$ \\
NuStar & 2012 Sep 19-25 & 56192.23 & $3000200600-2,3,5,7$ & 72.9 \\
\hline
\end{tabular}

\section{Introduction}

The X-ray spectrum of the accreting binary pulsar Her X-1 is characterized by a power law continuum with exponential cut-off and an apparent line-like feature, which was discovered in 1975 (Trümper et al. 1978). This feature is now generally accepted as an absorption feature around $40 \mathrm{keV}$ due to resonant scattering of photons off electrons on quantized energy levels (Landau levels) in the Teragauss magnetic field at the polar cap of the neutron star. The feature is therefore often referred to as a Cyclotron Resonant Scattering Feature (CRSF). The energy spacing between the Landau levels is given by $\mathrm{E}_{\mathrm{cyc}}=\hbar e B / m_{\mathrm{e}} c=11.6 \mathrm{keV} B_{12}$, where $B_{12}=B / 10^{12} \mathrm{G}$, providing a direct method of measuring the magnetic field strength at the site of the generation of the X-ray spectrum. The observed line energy is subject to gravitational redshift, $z$, such that the magnetic field may be estimated by $B_{12}=(1+\mathrm{z}) E_{\mathrm{obs}} / 11.6 \mathrm{keV}$. The discovery of the cyclotron feature in the spectrum of Her X-1 provided the first ever 'direct measurement' of the magnetic field strength of a neutron star, in the sense that no other model assumptions are needed. Originally considered an exception, cyclotron features are now known to be rather common in accreting X-ray pulsars, with more than a dozen binary pulsars being confirmed cyclotron line sources, with several objects showing multiple lines (up to four harmonics in $4 \mathrm{U} 0115+63$ ). Reviews are given by e.g. Coburn et al. 2002, Staubert 2003, Heindl et al. 2004, Terada et al. 2007, Wilms 2012.

Here we summarize some results, including our most recent ones, about the variability in cyclotron line energy. The application of pulse phase spectroscopy techniques has demonstrated a very strong variation in $\mathrm{E}_{\mathrm{cyc}}$ as function of pulse phase. Considering this pulse phase variation and the variation in pulse shape with $35 \mathrm{~d}$ phase, there must be a variation in the phase averaged $\mathrm{E}_{\text {cyc }}$ with $35 \mathrm{~d}$ phase. From a long-term study, we present the first statistically significant evidence for a true secular decrease of the phase averaged $\mathrm{E}_{\mathrm{cyc}}$. We speculate about the physics behind the secular decrease as being connected to changes in the configuration of the magnetic field structure at the polar caps of the accreting neutron star.

\section{Data base and method of analysis}

Her X-1 is probably the best observed accreting binary X-ray pulsar. Its X-ray spectrum and the cyclotron line (CRSF) has been measured by many instruments since the discovery of the CRSF 
Table 2: Recent cyclotron line energy measurements in Her X-1 by INTEGRAL, RXTE, Suzaku and NuStar. Uncertainties are at the $68 \%$ level. The two Suzaku data points from 2005 and 2006 are from Enoto et al. (2008), adjusted to describing the cyclotron line by a Gaussian line profile (see text). The NuStar value, as provided by the NuStar team, is preliminary. $35 \mathrm{~d}$ cycle numbering is according to Staubert et al. (1983, 2009a).

\begin{tabular}{llllll}
\hline Satellite & $\begin{array}{l}\text { Observation } \\
\text { month/year }\end{array}$ & $\begin{array}{l}\text { 35 d } \\
\text { cycle }\end{array}$ & $\begin{array}{l}\text { Center } \\
\text { [MJD] }\end{array}$ & $\begin{array}{l}\text { Cyclotron Line } \\
\text { Energy [keV] }\end{array}$ & $\begin{array}{l}\text { max. Flux } \\
{[\text { ASM cts/s] }}\end{array}$ \\
\hline RXTE & Feb 2009 & 388 & 54866.60 & $37.58 \pm 0.65$ & $5.94 \pm 0.50$ \\
Suzaku & May 2005 & 353 & 53648.90 & $38.70 \pm 1.00$ & $4.80 \pm 0.35$ \\
& Mar 2006 & 358 & 53823.90 & $38.10 \pm 1.00$ & $4.70 \pm 0.35$ \\
& Sep 2010 & 405 & 55461.60 & $37.80 \pm 0.34$ & $7.56 \pm 0.23$ \\
\multirow{4}{*}{ INTEGRAL L } & Sep 2012 & 426 & 56192.23 & not yet analysed & $6.94 \pm 0.20^{*}$ \\
& July/Aug 2007 & 373 & 54348.00 & $36.50 \pm 0.60$ & $4.50 \pm 0.48$ \\
& July 2010 & 403 & 55389.30 & $38.00 \pm 0.60$ & $8.50 \pm 0.24$ \\
NuStar & June 2011 & 413 & 55738.47 & $37.34 \pm 0.28$ & $7.00 \pm 0.20$ \\
& Sep 2012 & 426 & 56192.23 & $37.30 \pm 0.15$ & $6.94 \pm 0.20^{*}$ \\
\hline
\end{tabular}

* The maximum Main-On flux was determined using the monitoring data of RXTE/ASM and Swift/BAT (since 2012 from BAT only); the conversion is: $(2-10 \mathrm{keV} \mathrm{ASM} \mathrm{cts/s)}=90 *(15-$ $50 \mathrm{keV}$ BAT cts $\mathrm{cm}^{-2} \mathrm{~s}^{-1}$ )

in 1975 (Trümper et al. 1978). The data base behind previously reported results are summarized in corresponding Tables of the following publications: Gruber et al. 2001, Coburn et al. 2002, Staubert et al. (2007, 2009a, 2013), Klochkov et al. (2008, 2011) and Vasco et al. (2011, 2013). Details about more recent observations (proposed by our group during the last five years) by $R X T E$, INTEGRAL, Suzaku and NuStar are given in Tables 1 and 2. Generally, Main-On state observations $(35$ day-phases $<0.22$ ) were used. The spectral analysis was performed using the standard software appropriate for the respective satellites (see the publications cited above). Unless otherwise stated, the spectral model used was a power law with exponential cut-off (highecut, cutoffpl, Fermidirac) for the continuum and a multiplicative absorption line with a Gaussian optical depth profile for the cyclotron line. Details of the fitting procedure can be found in the papers cited above. Here we discuss results of the spectral analyses of pulse phase resolved and pulse phase averaged spectra, a dependence on 35 phase and on elapsed time.

\section{Variation of the cyclotron line energy $\mathbf{E}_{\mathrm{cyc}}$}

We will discuss the variability in the energy of the Cyclotron Resonant Scattering Feature (CRSF) in Her X-1 with regard to the following variables:

- Variation with the current X-ray luminosity, both on long and on short time scales

(the $35 \mathrm{~d}$ precessional period and the $1.24 \mathrm{~s}$ pulse period, respectively).

- Variation with phase of the $\mathbf{1 . 2 4} \mathrm{s}$ pulsation.

- Variation with phase of the $35 \mathrm{~d}$ precessional period.

- Variation with time, that is a true secular decay. 

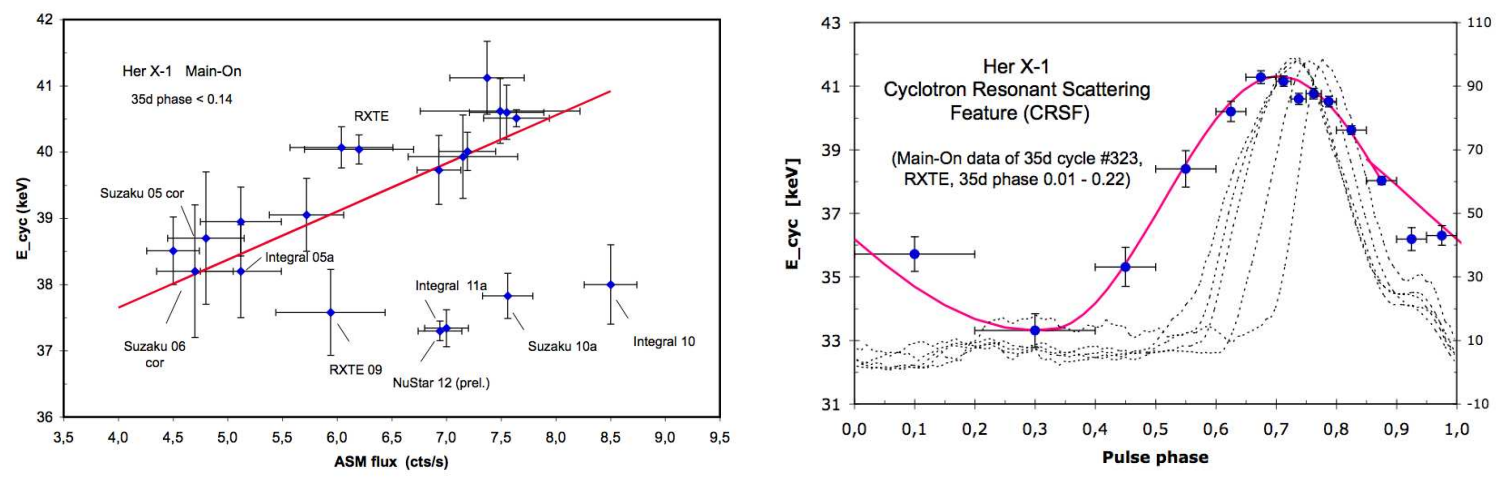

Figure 1: Left: The positive correlation between the cyclotron line energy and the maximum X-ray flux of the corresponding 35 day cycle as measured by RXTE/ASM (see Fig. 2 of Staubert et al., 2007) with eight added points: INTEGRAL 2005 (Klochkov et al. 2008), Suzaku of 2005 and 2006 (Enoto et al. 2008), RXTE 2009, INTEGRAL 2010, Suzaku 2010 and NuStar 2012 (preliminary). The solid red line is a linear fit, defining a slope of $(0.47 \pm 0.11) \mathrm{keV} /(\mathrm{ASM}$ cts/s). The Suzaku points of 2005/2006 have been 'corrected' upward by $2.8 \mathrm{keV}$, to account for the difference arising from the fact that in the analysis by Enoto et al. (2008) the Lorentzian profile was used, while for all others the Gaussian profile was used.

Right: Mean dependence of cyclotron line energy on pulse phase for the Main-On of $35 \mathrm{~d}$ cycle 323, as observed by RXTE/PCA in 2002 November. The solid red line represents a best fit function (a combination of two cosine components). The dotted lines are pulse profiles in the $30-45 \mathrm{keV}$ range for five different $35 \mathrm{~d}$ phases: $0.048,0.116,0.166,0.21$ and 0.24 . The main pulse is progressively moving to the right.

\subsection{Variation of $\mathbf{E}_{\mathrm{cyc}}$ with luminosity}

For Her X-1 the dependence of the centroid energy of the phase averaged cyclotron line on X-ray flux was discovered by Staubert et al. (2007) while analyzing an extended set of observations from RXTE. As reference X-ray flux the maximum of the 2-10 keV flux of the respective $35 \mathrm{~d}$ cycle as measured by the All Sky Monitor (ASM) on RXTE was used. The correlation was found to be positive, that is the cyclotron line energy $\mathrm{E}_{\mathrm{cyc}}$ increases with increasing $\mathrm{X}$-ray luminosity $L_{x}$.

The opposite behavior, a negative correlation (a decrease of $\mathrm{E}_{\mathrm{cyc}}$ with increasing $L_{x}$ ), had first been noted by Mihara (1995) in observations of a few high luminosity transient sources (4U 0115+63, Cep X-4, and V 0332+53) by Ginga. This negative correlation was associated with the high accretion rate during the X-ray outbursts, as due to a change in height of the shock (and emission) region above the surface of the neutron star with changing mass accretion rate, $\dot{M}$. In the model of Burnard et al. (1991), the height of the polar accretion structure is tied to $\dot{M}$. From this model one expects that an increase in accretion rate leads to an increase in the height of the scattering region above the neutron star surface, and therefore to a decrease in magnetic field strength and hence $\mathrm{E}_{\mathrm{cyc}}$. During the 2004/2005 outburst of V 0332+53 a clear anti-correlation of the line position with X-ray flux was observed (Tsygankov et al., 2006). The case of 4U 0115+63, however, is doubtful: while both Nakajima et al. (2006) and Tsygankov et al. (2007) had found a general anti-correlation between $\mathrm{E}_{\mathrm{cyc}}$ and luminosity in RXTE data of the Feb-Apr 1999 outburst, Müller, S. et al. (2012), analyzing RXTE and INTEGRAL data from an outburst in March/April 2008, have found that the negative correlation for the fundamental cyclotron line is likely an artifact due to correlations between continuum and line parameters when using the NPEX continuum model. 
The positive correlation in Her X-1 was secured by a re-analysis of the same RXTE data by Vasco et al. (2011), using the bolometric X-ray flux as reference. This analysis confirmed that the originally used $2-10 \mathrm{keV}$ flux is a good measure of the bolometric luminosity. While the above discussed analysis tests the correlated variability of $\mathrm{E}_{\mathrm{cyc}}$ and $L_{x}$ on long time scales ( $35 \mathrm{~d}$ and longer), the "pulse-amplitude resolved" analysis of Klochkov et al. (2011) does so on short time scales (down to the pulse period of $1.24 \mathrm{~s}$ ). Selecting pulses with amplitudes in certain ranges and producing mean spectra for each pulse amplitude range, showed that the cyclotron line energy scales positively with the mean pulse amplitude. In addition, it was found that the photon index $\Gamma$ of the underlying power law continuum scales negatively with the pulse amplitude. The same behavior was seen in data of the transient A $0535+26$. A recent pulse phase resolved analysis of A $0535+26$ observations by RXTE and INTEGRAL showed that data of one of the two peaks (of the double peak pulse profile) displays the same trend while data of the other peak do not (Müller, D. et al., 2013). Applying the same "pulse-amplitude resolved" technique to data of V 0332+53 and $4 \mathrm{U} 0115+63$, Klochkov et al. (2011) found the same behavior as originally detected in data sets that were selected on much longer time scales: $\mathrm{E}_{\mathrm{cyc}}$ decreases and $\Gamma$ increases with increasing $L_{x}$. Finally, we mention that a positive correlation of $\mathrm{E}_{\mathrm{cyc}}$ with $L_{x}$ was also seen by INTEGRAL in a flare of GX 304-1 (Klochkov et al., 2012).

Our current understanding of the physics behind these correlations assumes that we can distinguish between two accretion regimes in the accretion column above the polar cap of the neutron star: super- and sub-Eddington accretion. The former is responsible for the first detected negative correlation in high luminosity outbursts of transient X-ray sources: with increasing accretion rate $\dot{M}$, the shock and the scattering region move to larger height above the surface of the neutron star and consequently to weaker B-field (Burnard et al. 1991). Sub-Eddigton accretion, on the other hand, leads to the opposite behavior. In this regime an increasing $\dot{M}$ leads to an increased ram pressure on the scattering region and a resulting "squeezing" to smaller height and stronger B-field (Staubert et al., 2007). More detailed physical considerations have recently been presented by Becker et al. (2012). The persistent source Her X-1 is clearly a sub-Eddington source.

Fig. 1(left) reproduces the original correlation graph of Staubert et al. (2007) with new data points added (see Table 2). The $\mathrm{E}_{\mathrm{cyc}}$ values measured later than 2007 are significantly lower than the earlier values, while they maintain a similar positive slope. It is these points that establish the secular decay of $\mathrm{E}_{\mathrm{cyc}}$ which will be discussed below.

\subsection{Variation of $\mathbf{E}_{\mathrm{cyc}}$ with pulse phase}

Recently, Vasco et al. (2013) have presented results of pulse phase resolved analysis of RXTE observations. Fig. 1(right) shows the mean dependence of the centroid cyclotron line energy on pulse phase for all Main-On data of cycle 323 (for $35 \mathrm{~d}$ phases between 0.1 and 0.22 ). It is the first time that for Her X-1 a phase profile of the centroid cyclotron line energy $\mathrm{E}_{\mathrm{cyc}}$ is produced with such a high resolution and statistical accuracy (the four smallest bins around the peak of the pulse have a width of $1 / 80$ of a phase). The shape of the modulation is nearly sinusoidal, but for a good fit, at least two sine functions are needed (solid red line). The peak to peak amplitude of the modulation is $\sim 21 \%$ of the mean energy. There is no $35 \mathrm{~d}$ dependence of the shape of this modulation. Generally, the $\mathrm{E}_{\mathrm{cyc}}$ profile roughly follows the shape of the pulse profile, with its broad maximum close to the maximum of the main peak (pulse phases $0.7-0.8$ ). The dashed curves 
are $30-45 \mathrm{keV}$ pulse profiles for five different $35 \mathrm{~d}$ phases: the main peak is progressively moving to the right with increasing $35 \mathrm{~d}$ phases. ${ }^{1}$ The fact that the pulse moves, while the shape of the $\mathrm{E}_{\mathrm{cyc}}$ profile stays constant, leads to the expectation for a variation in pulse phase averaged $E_{c y c}$ with $35 \mathrm{~d}$ phase (see Section 3.3).

Pulse phase modulation in $\mathrm{E}_{\mathrm{cyc}}$ is quite common among accreting $\mathrm{X}$-ray pulsars and is generally believed to be due to the changing viewing angle under which the X-ray emitting regions are seen. If we adopt the idea, that peaks in the pulse profile can be associated with beamed radiation emitted from the accreting regions at the magnetic poles, then the observed pulse profiles are a representation of the emission characteristics. During a full neutron star rotation we may be seeing the beamed radiation of different emitting spots under changing angles. In addition, variable absorption and reflection at the inner edge of the precessing accretion disk and possibly a variable orientation due to precession of the neutron star (Postnov 2004) may contribute. The situation can be further complicated by gravitational bending. It is therefore not straightforward to interpret the observed pulse profiles and the variation in $\mathrm{E}_{\mathrm{cyc}}$ in terms of accretion geometry and beaming characteristics. We note here, that also the photon index $\Gamma$ of the power law continuum is dependent on pulse phase with a distinct minimum close to the pulse peak (Vasco et al., 2013).

\subsection{Variation of $\mathbf{E}_{\mathrm{cyc}}$ with precessional phase}

A few measured low $\mathrm{E}_{\mathrm{cyc}}$ values at late $35 \mathrm{~d}$ phases had led us to suspect that the pulse phase averaged cyclotron line energy may not be constant over the $35 \mathrm{~d}$ cycle. This has triggered proposals for repeated observations of Her X-1 at different $35 \mathrm{~d}$ phases by INTEGRAL, Suzaku and most recently NuStar. These observations are not yet fully analyzed, but using data from earlier RXTE observations (cycle 323), we have already clear indications, that there is a weak modulation of $\mathrm{E}_{\mathrm{cyc}}$ over the Main-On. Vasco et al. (2013) had shown, that the shape of the ( $\mathrm{E}_{\mathrm{cyc}} \mathrm{vs}$ pulse phase)-profile is not dependent on $35 \mathrm{~d}$ phase, while the pulse profile itself is. Fig. 1(right) demonstrates that the $30-45 \mathrm{keV}$ pulse profiles (this is the energy range which includes most of the CRSF) clearly move to the right with increasing $35 \mathrm{~d}$ phase, which means that more and more photons are found at pulse phases where the cyclotron line energy is decreasing. So, a modulation of $\mathrm{E}_{\mathrm{cyc}}$ with $35 \mathrm{~d}$ phase is inevitable. In order to quantitatively test this, we have performed a formal folding of $30-45 \mathrm{keV}$ pulse profiles (for 10 different $35 \mathrm{~d}$ phases) with template ( $\mathrm{E}_{\mathrm{cyc}}$ vs pulse phase)-profiles at the same $35 \mathrm{~d}$ phases. These template profiles were constructed by inter- and extrapolation of the four individual profiles at different $35 \mathrm{~d}$ phases given in Fig. 3 and 4 of Vasco et al. (2013), taking into account that both the maximum value and the peak-to-peak amplitude are slightly $35 \mathrm{~d}$ phase dependent. By folding the pulse profile with the corresponding $\mathrm{E}_{\mathrm{cyc}}$ profile, the expected pulse phase averaged $\mathrm{E}_{\mathrm{cyc}}$ value can be calculated. In Fig. 2(left) these calculated values are shown as blue triangles (connected by the solid blue line): a slow increase up to phase $\sim 0.19$ is followed by a somewhat sharper decay. For comparison, we show the directly measured phase averaged $\mathrm{E}_{\mathrm{cyc}}$ values (data points with uncertainties) for 13 small integration intervals covering the Main-On of cycle 323 . The directly measured values are consistent with the calculated modulation. With the final analysis of additional data, more definite conclusions are expected. This new result

\footnotetext{
${ }^{1}$ The definition of pulse phase zero is tied to the "sharp edge" at the decay of the right shoulder of the main peak (see Staubert et al., 2009b, 2013).
} 

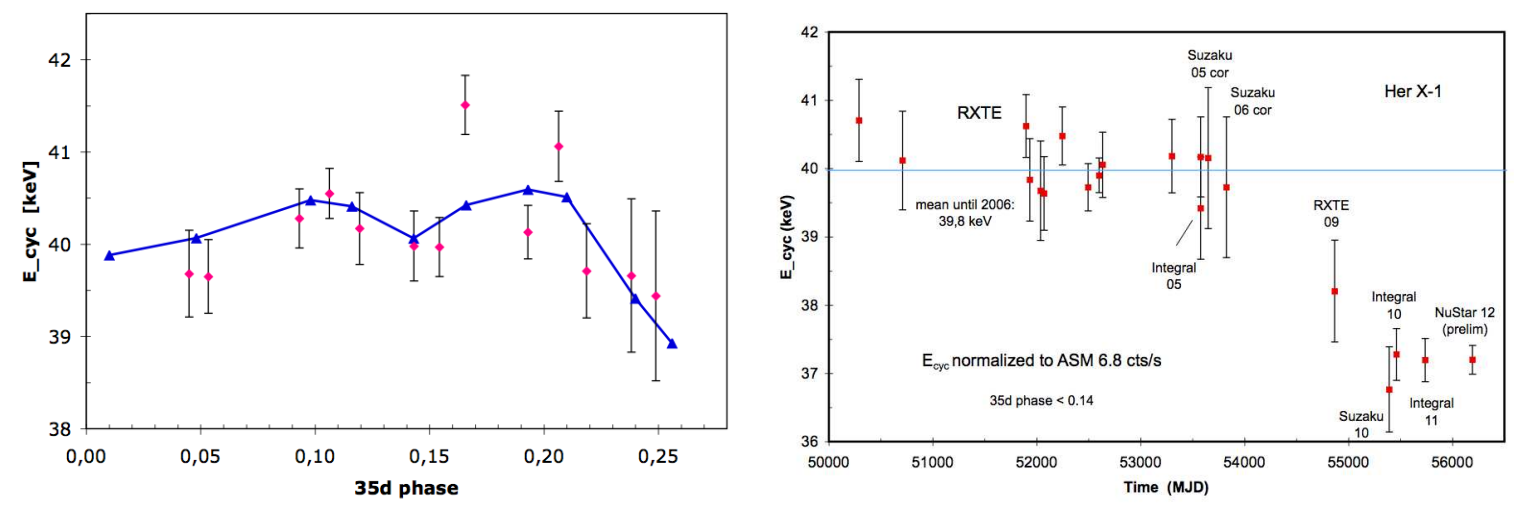

Figure 2: Left: Centroid pulse phase averaged cyclotron line energies at different $35 \mathrm{~d}$ phases of Main-On cycle 323. The data points with uncertainties are direct measurements for ten small integration intervals. The blue triangles connected by the solid blue line are values which are calculated by folding observed pulse profiles in the $30-45 \mathrm{keV}$ range with template ( $\mathrm{E}_{\mathrm{cyc}}$ vs pulse phase)-profiles for the same $35 \mathrm{~d}$ phases.

Right: Her X-1 pulse phase averaged cyclotron line energies $\mathrm{E}_{\mathrm{cyc}}$, normalized to a reference ASM count rate of $6.9 \mathrm{cts} / \mathrm{s}$ using the originally found correlation function. A break in $\mathrm{E}_{\mathrm{cyc}}$ is apparent around MJD 54000. Note, that the four points measured last are all low, indicating a true secular variation.

adds another piece to the puzzle on the question about the physical nature of the $35 \mathrm{~d}$ modulation - precession of the accretion disk (and?) precession of the neutron star? - and the mechanism of generating the varying pulse profiles and the varying spectra - CRSF and continua (see discussion in Staubert et al., 2009a, 2013 and Vasco et al., 2013).

\subsection{Variation of $\mathbf{E}_{\mathrm{cyc}}$ with time - secular variation}

In investigating the long-term behavior of the pulse phase averaged centroid cyclotron line energy, Staubert et al. (2007) had discovered that $\mathrm{E}_{\mathrm{cyc}}$ depends on the X-ray flux of the source. Their Fig. 1 compiles the historical measurements until this time: after the initial measurements in the range 34-37 keV, a (so far unexplained) upward jump occurred, between 1991 and 1994, to beyond $42 \mathrm{keV}$, followed by an apparent decrease with time. This decrease had been noticed after the first observations with RXTE in 1996 and 1997 (in comparison to values from CGRO/BATSE and Beppo/SAX), and had served as an important argument to ask for more observations of Her X1. But then, Staubert et al. (2007) showed that this decrease with time was largely an artifact due to the dependence of $\mathrm{E}_{\mathrm{cyc}}$ on X-ray flux. Plotting $E_{\text {cyc }}$ against the 2-10 keV X-ray flux, as measured by ASM on RXTE shows a clear positive correlation, with an increase in $\mathrm{E}_{\text {cyc }}$ by $\sim 7 \%$ for a factor of two increase in flux (see Section 3.1). So, here nature had conspired such that later measurements were (on average) taken when the flux happened to be low (Her X-1 is known for varying its flux within a factor of two, on time scales of a few $35 \mathrm{~d}$ cycles).

Now, however, the situation has changed. Here, we report about the first statistically significant evidence for a true secular decay of the phase averaged cyclotron line energy. From Fig. 1(left), it is already evident that $E_{\text {cyc }}$ values measured after 2008 are significantly lower, while maintaining a similar positive correlation within themselves (possibly less steep). In order to separate the dependence on time and the dependence on $\mathrm{X}$-ray flux, we have normalized the measured $\mathrm{E}_{\mathrm{cyc}}$ values to 
a common X-ray flux (namely 6.8 ASM cts/s), using the slope as found in the original flux correlation. The result is shown in Fig 2(right). The normalized $\mathrm{E}_{\mathrm{cyc}}$ values until 2006 are consistent with a constant value of $39.8 \mathrm{keV}$, a significant decrease occurs after that. This decrease is consistent with a linear decay with time between 2006 and 2012, but a more abrupt change can not be excluded. We note, that all measurements used for this plot are from observations corresponding to the Main-On of Her X-1 at $35 \mathrm{~d}$ phases less than 0.14 (for which the dependence on $35 \mathrm{~d}$ phase is very weak).

With regard to the physical implications of the now observed secular decrease of the cyclotron line energy, we speculate that the phenomenon could be connected to a restructuring of the polar magnetic field of the neutron star. This may be due to an adjustment of the field when coping with the continued accretion of plasma. Theoretically, such scenarios have been investigated e.g., by Brown and Bildsten (1998), Litwin et al. (2001), Mukherjee and Bhattacharya (2012) and Mukherjee et al. (2012).

\section{References}

[1] Becker P.A., Klochkov D., Schönherr G., Wilms J., Staubert R. et al. 2012, A\&A, 544, A123

[2] Brown E. F. and Bildsten L. 1998, ApJ, 496, 915

[3] Burnard D. J., Arons J., Klein R. I. 1991, ApJ, 762, 61

[4] Coburn W., Heindl W. A., Rothschild R. E., Gruber D. E., Kreykenbohm I. et al. 2002, ApJ, 580, 394

[5] Enoto T., Makishima K., Terada Y., Mihara, T., Nakazawa, K. et al. 2008, PASJ, 60, 57

[6] Heindl W., Rothschild R. E., Coburn W., Staubert R., Willms J. et al. 2004, in Proc. "X-ray Timing 2003: Rossi and Beyond", AIP 714, p.323

[7] Klochkov D., Staubert R., Postnov K. A., Shakura N. I., Santangelo, A. et al. 2008, A\&A, 482, 907

[8] Klochkov D., Staubert R., Santangelo A., Rothschild R. E., Ferrigno C. 2011, A\&A, 532, A126

[9] Klochkov D., Doroshenko V., Santangelo A., Staubert R., Ferrigno C. et al. 2012, A\&A, 542, L28

[10] Litwin C., Brown E. F., and Rosner R. 2001, ApJ, 553, 788

[11] Mihara T. 1995, PhD Thesis, Univ. of Tokyo

[12] Müller S., Ferrigno C., Kühnel M., Schönherr G. et al. 2012, A\&A, in print, astro-ph.HE 1211.6298

[13] Müller D., Klochkov D., Caballero I., Staubert R., Santangelo A. 2013, PoS(INTEGRAL12), this Vol.

[14] Mukherjee D. and Bhattacharya D. 2012, MNRAS, 420, 720

[15] Mukherjee D., Bhattacharya D., Mignone A. 2012, MNRAS, in print, astro-ph.HE1212.3897

[16] Nakajima M., Mihara T., Makishima K., Niko, H. 2006, ApJ, 646, 1125

[17] Postnov K. 2004, http://astro.uni-tuebingen.de/staubert65/talks/postnov.pdf

[18] Staubert R., Bezler M., Kendziorra E. 1983, A\&A, 117, 215

[19] Staubert R. 2003, in "Multifrequency behaviour of high energy cosmic sources", ChJAA, 3, S270

[20] Staubert R., Shakura N. I., Postnov K., Wilms, J., Klochkov D. et al. 2007, A\&A, 465, L25

[21] Staubert R., Klochkov D., Postnov K., Shakura N., Willms J., Rothschild R. 2009a, A\&A 494, 1025

[22] Staubert R., Klochkov D., Wilms J. 2009b, $A \& A, \mathbf{5 0 0}, 883$

[23] Staubert R., Vasco, D., Klochkov D., Wilms J. 2013, A\&A, 550, A110 (astro-ph.HE 1212.5472)

[24] Terada Y. et al. 2007, in Adv. Space Res. 40, 1485

[25] Trümper J., Pietsch W., Reppin, C., Voges W., Staubert R., Kendziorra E. 1978, ApJ 219, L105

[26] Tsygankov S. S., Lutovinov A. A., Churazov E. M., Sunyaev R. A. 2006, MNRAS, 371, 19

[27] Tsygankov S. S., Lutovinov A. A., Churazov E. M., Sunyaev R. A. 2007, Astr. Lett., 33, 368

[28] Vasco D., Klochkov D., Staubert R. 2011, A\&A 532, A99

[29] Vasco D., Staubert, R., Klochkov D., Santangelo A. et al. 2013, A\&A, 550, A111

[30] Wilms J. 2012, in Proceed. 39th COSPAR Scientific Assembly, 14-22 July 2012, in Mysore, India: "Cyclotron Lines of Accreting Neutron Stars", Vol. 39, p. 2159 